\title{
Exposure to low-dose nanopolystyrene induces the response of neuronal JNK MAPK signaling pathway in nematode Caenorhabditis elegans
}

\author{
Man Qu, Dan Li, Yunli Zhao, Yujie Yuan and Dayong Wang ${ }^{*}$
}

\begin{abstract}
Background: The response of organisms to nanoplastic exposure has gradually received the attention. Nevertheless, the role of neurons in response to nanoplastic exposure and the underlying mechanism are still largely unclear. We here examined the role of neuronal JNK MAPK signaling in response to low-dose of polystyrene $(100 \mathrm{~nm})$ in Caenorhabditis elegans.

Results: Exposure to nanopolystyrene in the range of $\mu \mathrm{g} / \mathrm{L}$ could increase the expression of genes $(j k k-1$, mek-1, and jnk-1) encoding JNK MAPK signaling pathway. Meanwhile, RNAi knockdown of any of these genes induced a susceptibility to nanopolystyrene toxicity. In the neurons, SNB-1/synaptobrevin was identified as the downstream target of JNK-1/JNK, suggesting the alteration in neurotransmitter signals in nanopolystyrene-exposed nematodes. In nanopolystyrene-exposed nematodes, JNK-1 modulated TBH-1-mediated octopamine signal and CAT-2-mediated dopamine signal. TBH-1 and CAT-2 further regulated the response to nanopolystyrene by affecting the function of corresponding intestinal octopamine receptors (SER-6 and OCTR-1) and intestinal dopamine receptor (DOP-1). In the intestine, DOP-1 regulated the response to nanopolystyrene by activating the downstream signaling cascade in p38 MAPK signaling pathway.

Conclusions: Exposure to low-dose of nanopolystyrene could induce the response of neuronal JNK MAPK signaling pathway in nematodes. Our data further highlight the crucial role of neuronal JNK MAPK signaling-activated alteration in octopamine and dopamine signals in regulating the response to nanopolystyrene in organisms.
\end{abstract}

Keywords: Nanopolystyrene, JNK MAPK signaling, Neuron, Caenorhabditis elegans

\section{Background}

A growing amount of reports have demonstrated that polymer microplastics $(\leq 5 \mathrm{~mm})$ have been frequently detected and widely distributed in different freshwater, marine, soil, and atmospheric environments, and even in the commodity salt [1-6]. Moreover, it has been found that the nanoplastics $(\leq 100 \mathrm{~nm})$, such as

\footnotetext{
*Correspondence: dayongw@seu.edu.cn

Key Laboratory of Environmental Medicine Engineering of Ministry of Education, Medical School, Southeast University, Nanjing 210009, China
}

nanopolystyrene, have been widely used in daily necessities, such as personal care products [7]. With the increase in production and use, a large amount of nanopolystyrene is potentially released into the environment. Meanwhile, microplastics in the real environment will also be gradually degraded into particles with nanosize [8]. Recently, the microplastics including nanoplastics have been frequently detected in Yangtze River basin, China $[3,9,10]$. Therefore, the toxicological responses of organisms to nanoplastics are needed to be examined.

The ecotoxicity at various aspects of nanopolystyrene exposure has been detected in different environmental 
organisms, such as monogonont rotifer and sea urchin [11-16]. Nanopolystyrene could not only be accumulated in exposed zebrafish, but also be transferred to the progeny [17]. Caenorhabditis elegans is a classic model animal, which was initially isolated from the soil environment [18]. Caenorhabditis elegans has been shown to be highly sensitive to the toxicity of environmental toxicants or stresses [19-21]. Some sublethal endpoints, such as brood size, locomotion behavior, and reactive oxygen species (ROS) production, have been raised for evaluating this sensitivity in nematodes [22-24]. Caenorhabditis elegans has been used for the assessment of both pristine and chemical modified nanopolystyrene particles in inducing toxicity at various aspects, such as reproductive toxicity and intestinal toxicity [25-28]. It has been predicted that the environmental concentrations of nanoplastics $(100 \mathrm{~nm})$ are in the range $\leq 1 \mu \mathrm{g} / \mathrm{L}[29,30]$. Caenorhabditis elegans is useful for detecting the potential toxicity of nanopolystyrene $(100 \mathrm{~nm})$ at predicted environmental concentration [31, 32]. In nematodes, some intestinal signaling pathways (such as insulin and p38 mitogen-activated protein kinase (MAPK) signaling pathways) are required for the response to nanopolystyrene [33-35]. Nevertheless, the roles of molecular signals in other tissues (such as neurons) in response to the nanopolystyrene are still largely unclear.

c-Jun N-terminal kinase (JNK) MAPK signaling pathway, one of the MAPK signaling family, acts as a central signaling hub to regulate various important biological processes by transducing extracellular cues into the cells $[36,37]$. In nematodes, the JNK MAPK signaling has been also proven to be involved in the regulation of stress response [38, 39]. For example, the JNK MAPK signaling pathway was involved in the regulation of graphene oxide (GO) toxicity [39]. The C. elegans JNK MAPK signaling pathway mainly contains JNK-1, homolog of human JNK, and two MAP kinase kinases (MEK-1 and JKK-1) [40]. MEK-1 and JKK-1 act as activators of JNK [40]. jnk-1 is expressed in the neurons [40]. In this study, we employed C. elegans as an animal model to investigate the response of neuronal JNK MAPK signaling to nanopolystyrene. Our results suggested the crucial role of JNK MAPK signaling pathway in response to nanopolystyrene. Moreover, our data highlight the important functions of octopamine and dopamine signals-mediated communication between neurons and intestine in regulating the response to nanopolystyrene in nematodes.

\section{Materials and methods}

\section{Physicochemical characterizations of nanopolystyrene}

Nanopolystyrene $(100 \mathrm{~nm})$ was from Janus New-Materials Co. (Nanjing, China). Working concentrations (0.1, 1,10 , and $100 \mu \mathrm{g} / \mathrm{L}$ ) of nanopolystyrene were prepared by diluting the stock solutions with liquid K-medium. Physicochemical properties of nanopolystyrene particles were examined by transmission electron microscopy (TEM, JEOL Ltd., Japan), Raman spectroscopy (Renishaw Invia Plus laser Raman spectrometer, Renishaw, UK), dynamic light scattering (DLS), and Zeta potential (Zetasizer Nano-ZS90, Malvern Instruments Ltd., UK). Before the use, the particle solutions were sonicated for $30 \mathrm{~min}$ $(40 \mathrm{kHz}, 100 \mathrm{~W})$.

\section{Animal maintenance}

All nematodes were maintained on normal nematode growth media (NGM) agar plates fed with a lawn of Escherichia coli OP50 as the food source [18]. To collect synchronous L1 larvae, gravid nematodes were lysed with a bleaching mixture solution $(0.45 \mathrm{M} \mathrm{NaOH}$ and $2 \%$ $\mathrm{HOCl})$ to release eggs from the body of animals. After that, the eggs were transferred to a new NGM plates to let them develop into the L1 larvae.

\section{Exposure and toxicity assessment}

Nanopolystyrene exposure was performed from L1 larvae to adult day-3 in liquid solutions with the addition of OP50 $\left(\sim 4 \times 10^{6}\right.$ colony-forming units (CFUs) $)$ [33]. The nanopolystyrene solutions were refreshed daily.

ROS production was used to reflect the activation of oxidative stress in nanopolystyrene-exposed nematodes [41]. After the nanopolystyrene exposure, the nematodes were incubated with $1 \mu \mathrm{M} \mathrm{CM}-\mathrm{H}_{2}$ DCFDA for $3 \mathrm{~h}$ without light. After the labeling, the nematodes were further washed with M9 buffer for three times. The nematodes were then transferred onto $2 \%$ agar pads for the examination at $488 \mathrm{~nm}$ of excitation wavelength and at $510 \mathrm{~nm}$ of emission filter with a laser scanning confocal microscope. In nematodes, the strongest ROS fluorescent signals can be detected in the intestine [42]. Fluorescence intensity in intestine was semi-quantified in comparison to the autofluorescence using Image J software. Fifty nematodes were examined per treatment.

Locomotion behaviors of head thrash and body bend were used as indicatives of functional state of motor neurons [43]. After the nanopolystyrene exposure, the nematodes were washed with M9 buffer for three times. To detect the number of head thrashes, 40 nematodes were randomly picked on the surface of an NGM plate without OP50 for the counting under a stereomicroscopy. A head thrash is defined as a change in the direction of bending at mid-body. Similarly, 40 nematodes were randomly picked onto an NGM plate without OP50 for the counting. A body bend is counted as a change in the direction of posterior bulb part along the $y$ axis, assuming that the nematodes were traveling along the $x$ axis. 


\section{Quantitative real-time polymerase chain reaction (qRT-PCR)}

Total RNA of nematodes in each group was isolated using Trizol (Sigma-Aldrich, St. Louis, MO, USA). RNA purity and concentration were guaranteed by the ratio of OD260/280 in a spectrophotometer. Mastercycler gradient PCR system (Eppendorf, USA) was employed to synthesize cDNA with reverse-transcriptase reaction. Expression levels of the examined genes were determined by StepOnePlus ${ }^{\mathrm{TM}}$ real-time PCR system (Applied Biosystems, Carlsbad, USA) with the SYBR Green qRT-PCR master mix (TOYOBO, Japan) and calculated using $2^{-\Delta \Delta C t}$ method. The $t b a-1$ encoding alpha-tubulin protein was selected as a reference gene, and comparative cycle threshold methods were used to determine the relative quantities of the examined genes. Three biological replicates were performed in all reactions. Primers information for $\mathrm{qRT}-\mathrm{PCR}$ is shown in Additional file 1: Table S1.

\section{RNA interference (RNAi) assay}

Before growing onto NGM agar plates, E. coli HT115 (DE3) was transferred into LA broth containing isopropyl 1-thio- $\beta$-D-galactopyranoside (IPTG, $5 \mathrm{mM}$ ). The L1 larvae were fed with HT115 (E. coli strain) carrying double-stranded RNA corresponding to jkk-1, mek-1, jnk-1, snb-1, octr-1, tyra-3, ser-6, dop-1, tbh-1, or cat2 [44]. Once the L1 larvae on RNAi plates became the gravid animals, they were picked on fresh RNAi plate to lay eggs. The second generation was used for exposure and toxicity assessment. HT115 bacteria harboring empty vector L4440 was used as a control. RNAi efficiency was checked by qRT-PCR (Additional file 1: Figure S1).

\section{DNA constructs and transformation}

The promoter of $u n c-14$ (specially expressed in neurons) was amplified by PCR from wild-type nematode genomic DNA. PCR-amplified jnk-1/B0478.1 was inserted into vector pPD_95_77 carrying the unc-14 promoter sequence. Germline transformation was conducted by co-injecting a testing DNA $(1040 \mu \mathrm{g} / \mathrm{mL})$ and a marker DNA of Pdop-1::rfp $(60 \mu \mathrm{g} / \mathrm{mL})$ into gonad [45]. Primers' information for vector constructions is shown in Additional file 1: Table S2.

\section{Statistical analysis}

Statistical analysis was carried out using SPSS Statistics 19.0 Software (SPSS Inc., USA.). After checking the normality using Agostino $D$ test before parameters statistics, differences between groups were tested using one-way analysis of variance (ANOVA). Two-way
ANOVA analysis was further performed for multiple factor comparison. Probability level of 0.01 was considered statistically significant.

\section{Results \\ Properties of nanopolystyrene}

The TEM data show the morphology and the size of nanopolystyrene (Additional file 1: Figure S2a). After the sonication, the nanopolystyrene in the range of $\mu \mathrm{g} / \mathrm{L}$ can be well dispersed in K medium [33]. The DLS analysis further confirmed that the size of nanopolystyrene after the sonication was $103.45 \pm 4.8 \mathrm{~nm}$. The Raman spectroscopy analysis indicated that the nanopolystyrene showed the peaks at $985.93 \mathrm{~cm}^{-1}$ (breathing vibration of benzene ring), at $1027.06 \mathrm{~cm}^{-1}$ and $1071.15 \mathrm{~cm}^{-1}$ (symmetric extension vibration of carbon atoms in benzene ring), at $1107.58 \mathrm{~cm}^{-1}, 1130.08 \mathrm{~cm}^{-1}, 1137.97 \mathrm{~cm}^{-1}$, and $1146.82 \mathrm{~cm}^{-1}$ (stretching vibration of carbon atoms between benzene ring and polyethylene group), at $1350.26 \mathrm{~cm}^{-1}$ (asymmetric stretching vibration of carbon atoms between benzene ring and polyethylene group), at $1475.36 \mathrm{~cm}^{-1}$ (asymmetric bending vibration of carbon atoms and hydrogen atoms), and at $1513.10 \mathrm{~cm}^{-1}$ (asymmetric stretching vibration of benzene ring carbon atoms) (Additional file 1: Figure S2b). The zeta potential of nanopolystyrene was $-9.213 \pm 0.478 \mathrm{mV}$.

\section{Effect of nanopolystyrene exposure on expressions of genes encoding JNK MAPK signaling pathway} After the exposure, nanopolystyrene $(0.1 \mu \mathrm{g} / \mathrm{L})$ did not affect the expressions of $j k k-1$, mek-1, and $j n k-1$, and nanopolystyrene $(1 \mu \mathrm{g} / \mathrm{L})$ also did not influence the expressions of $j k k-1$ and $m e k-1$ (Fig. 1a). In contrast, exposure to nanopolystyrene $(1 \mu \mathrm{g} / \mathrm{L})$ significantly increased the jnk-1 expression (Fig. 1a). Exposure to nanopolystyrene at concentrations of 10 and $100 \mu \mathrm{g} / \mathrm{L}$ further significantly increased the expressions of $j k k-1, m e k-1$, and $j n k-1$ (Fig. 1a).

\section{RNAi knockdown of $j k k-1$, mek-1, or jnk-1 induced a susceptibility to nanopolystyrene toxicity}

Using intestinal ROS production and locomotion behavior as endpoints, we observed the more severe induction of intestinal ROS production and decrease in locomotion behavior in nanopolystyrene-exposed $j k k-1$ (RNAi), mek-1(RNAi), or jnk-1(RNAi) nematodes compared with those in nanopolystyrene-exposed wild-type nematodes (Fig. 1b, c). That is, RNAi knockdown of $j k k-1, m e k-1$, or $j n k-1$ caused a susceptibility of nematodes to the nanopolystyrene toxicity. 


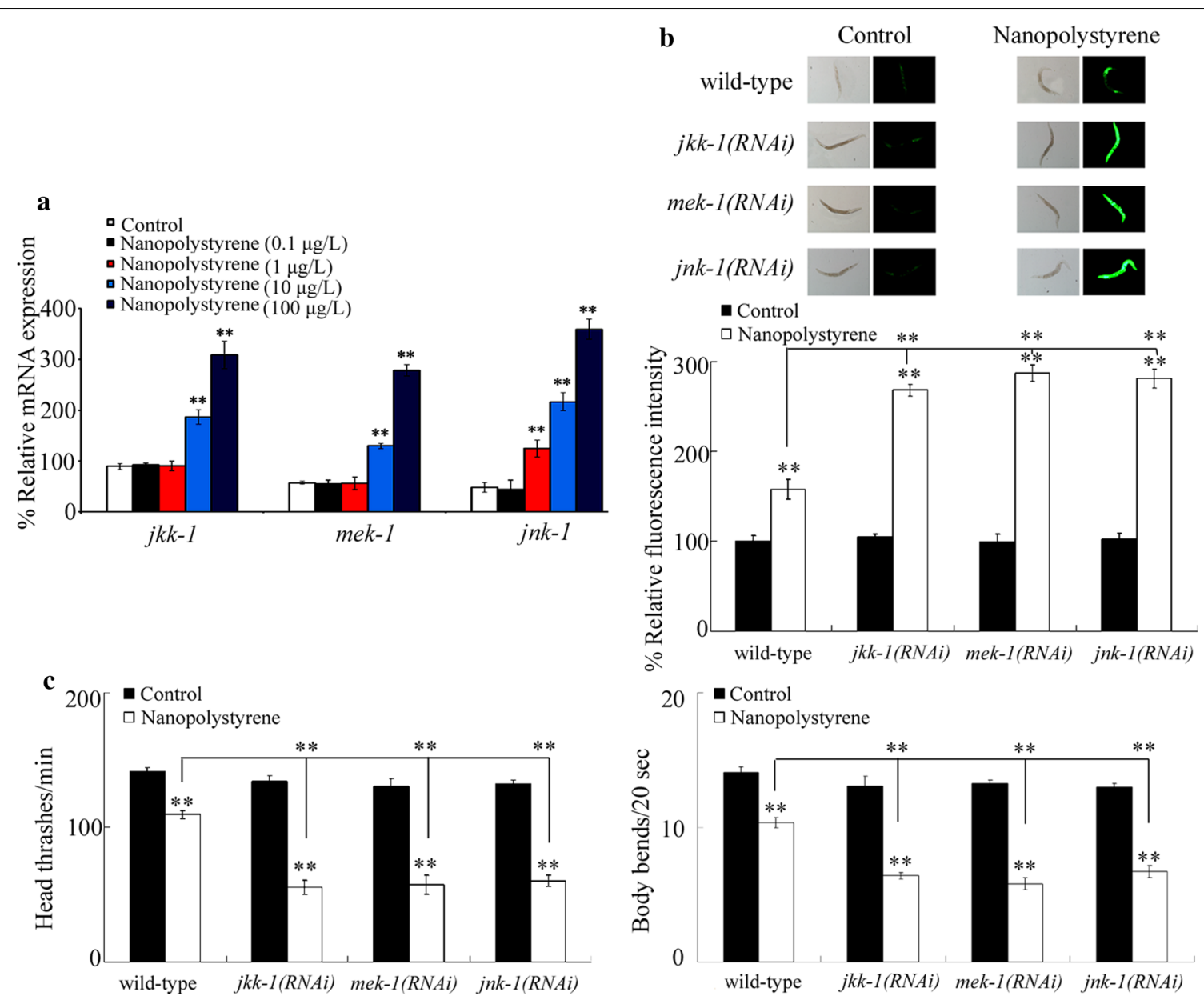

Fig. 1 Response of JNK MAPK signaling pathway to nanopolystyrene exposure in nematodes. a Effect of nanopolystyrene exposure on expression of genes encoding the JNK MAPK signaling pathway. Bars represent mean \pm SD. ${ }^{* *} P<0.01$ vs. control. b Effect of $j k k-1$, mek-1, or jnk-1 RNAi knockdown on nanopolystyrene toxicity in inducing intestinal ROS production. Exposure concentration of nanopolystyrene was $1 \mu \mathrm{g} / \mathrm{L}$. Bars represent mean $\pm S D$. ${ }^{* *}<0.01$ vs. control (if not specially indicated). c Effect of $j k k-1$, mek-1, or jnk-1 RNAi knockdown on nanopolystyrene toxicity in decreasing locomotion behavior. Exposure concentration of nanopolystyrene was $1 \mu \mathrm{g} / \mathrm{L}$. Bars represent mean $\pm S D$. ${ }^{* *} P<0.01 \mathrm{vs}$. control (if not specially indicated). Exposure was performed from L1 larvae to adult day-3

\section{Identification of targets for JNK-1 in regulating the response to nanopolystyrene}

Previous studies have raised some potential downstream neuronal targets (such as UNC-16, SHC-1, and SNB-1) of JNK-1 [46, 47]. UNC-16 is a JNK-signaling scaffold protein, SHC-1 is a p52Shc, and SNB-1 is a synaptobrevin. Exposure to nanopolystyrene did not alter expressions of $u n c-16$ and $s h c-1$ (Additional file 1: Figure S3a). Different from this, nanopolystyrene exposure significantly increased the $s n b-1$ expression (Additional file 1: Figure S3a). In nematodes, jnk-1 encodes a neuronal protein [40]. Meanwhile, in nanopolystyrene-exposed nematodes, RNAi knockdown of jnk-1 could significantly decrease the $s n b-1$ expression (Additional file 1: Figure S3b). In nematodes, we observed the more severe induction of intestinal ROS production and decrease in locomotion behavior in nanopolystyrene-exposed $s n b$ 1(RNAi) nematodes compared with those in nanopolystyrene-exposed wild-type nematodes (Additional file 1: Figure S3c and Additional file 1: Figure S4a). These results suggested the potential role of SNB-1 as the downstream target of neuronal JNK-1 in regulating the response to nanopolystyrene.

\section{SNB-1 acted downstream of neuronal JNK-1 to regulate the response to nanopolystyrene}

To determine the genetic interaction between SNB-1 and JNK-1 in regulating the response to nanopolystyrene, we generated transgenic strain Is(Punc-14-jnk-1) overexpressing neuronal JNK-1. Neuronal overexpression of 
JNK-1 could prevent the toxicity of nanopolystyrene in inducing intestinal ROS production and in decreasing locomotion behavior (Additional file 1: Figures S3d and S4b), suggesting the resistance of nematodes with neuronal overexpression of JNK-1 to nanopolystyrene toxicity. Moreover, we observed that RNAi knockdown of snb-1 could induce the significant induction of intestinal ROS production and decrease in locomotion behavior in nanopolystyrene-exposed nematodes overexpressing neuronal JNK-1 (Additional file 1: Figures S3d and S4b). That is, RNAi knockdown of $s n b-1$ could suppress the resistance of nematodes overexpressing neuronal JNK-1 to nanopolystyrene toxicity. Therefore, SNB-1 acted as a downstream target of neuronal JNK-1 to regulate the response to nanopolystyrene.

\section{Involvement of intestinal neurotransmitter receptors of OCTR-1, SER-6, and DOP-1 in regulating the response to nanopolystyrene}

SNB-1/synaptobrevin is a vesicle-associated protein, which mediates the biological process of neurotransmitter release [48]. After the exposure, a large amount of nanopolystyrene particles were translocated and accumulated in the intestinal cells [49]. We assumed that the SNB-1-mediated neuronal neurotransmission may regulate the nanopolystyrene toxicity by activating the corresponding intestinal neurotransmitter receptors. Among the neurotransmitter receptors in nematodes, 2 octopamine receptors (OCTR-1 and SER-6), 1 tyramine receptor (TYRA-3), 3 serotonin receptors (SER-1, SER-3, and SER-5), 3 acetylcholine receptors (ACR-9, ACR-14, and ACR-17), and 2 dopamine receptors (DOP-1 and DOP-4) can be expressed in the intestinal cells (Additional file 1: Table S3). Among the genes encoding these intestinal neurotransmitter receptors, exposure to nanopolystyrene could significantly decrease the expression of octr-1 and increase the expressions of tyra-3, ser-6, and dop-1 (Fig. 2a). Using VP303/rde-1(ne219);kbIs7[nhx$2 p:: r d e-1+$ rol-6(su1006)] as a genetic tool for intestinespecific RNAi knockdown of gene(s) [50], we found that intestine-specific RNAi knockdown of tyra-3 did not significantly affect the nanopolystyrene toxicity in inducing intestinal ROS production (Fig. 2b). In contrast, intestine-specific RNAi knockdown of octr-1 suppressed the nanopolystyrene toxicity in inducing intestinal ROS production, and intestine-specific RNAi knockdown of ser- 6 or dop-1 caused the more severe induction of intestinal ROS production in nanopolystyrene-exposed nematodes compared with that in nanopolystyrene-exposed VP303 nematodes (Fig. 2b). These observations suggested that

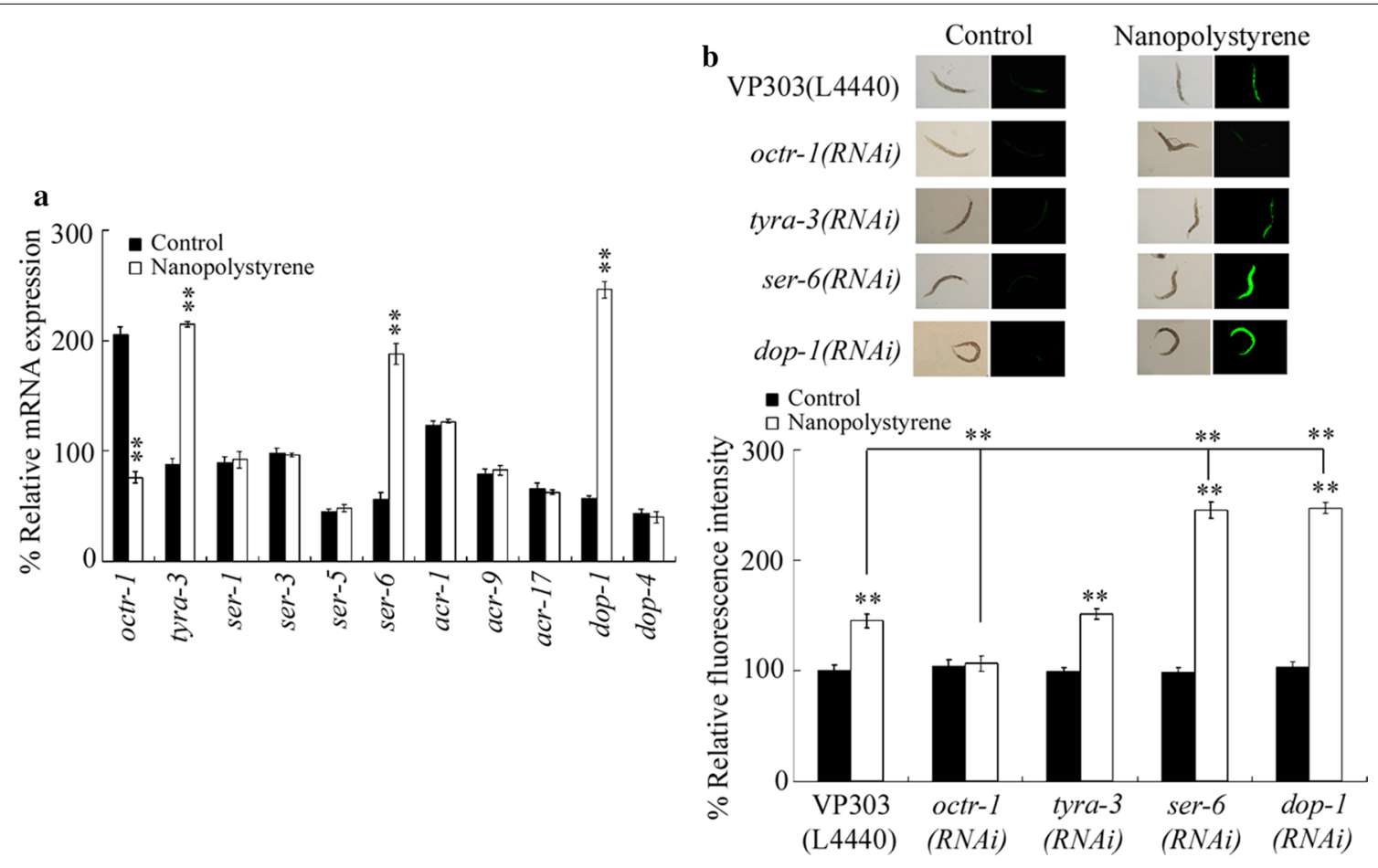

Fig. 2 Identification of intestinal neurotransmitter receptors involved in the regulation of response to nanopolystyrene. a Effect of nanopolystyrene exposure on expressions of genes encoding intestinal neurotransmitter receptors. Bars represent mean $\pm S D$. ${ }^{* *} P<0.01$ vs. control. $\mathbf{b}$ Effect of octr-1, tyra-3, ser-6, or dop-1 RNAi knockdown on nanopolystyrene toxicity in inducing intestinal ROS production. Bars represent mean $\pm S D .{ }^{* *} P<0.01$ vs. control (if not specially indicated). Exposure concentration of nanopolystyrene was $1 \mu \mathrm{g} / \mathrm{L}$. Exposure was performed from L1 larvae to adult day-3 
intestinal octopamine receptors (OCTR-1 and SER-6) and dopamine receptor (DOP-1) were involved in the control of response to nanopolystyrene.

\section{Involvement of TBH-1 and CAT-2 in the control of response to nanopolystyrene}

In nematodes, the octopamine biosynthesis requires tyramine $\beta$-hydroxylase $\mathrm{TBH}-1$ to convert tyramine into octopamine [51], and the dopamine biosynthesis requires tyrosine hydroxylase CAT-2 [52]. Nanopolystyrene exposure could significantly increase the $t b h-1$ expression and decrease the cat-2 expression (Fig. 3a). Meanwhile, RNAi knockdown of jnk-1 could cause the significant decrease in tbh-1 expression and increase in cat-2 expression in nanopolystyrene-exposed nematodes (Fig. 3b). Moreover, we observed the more severe induction of intestinal ROS production and decrease in locomotion behavior in nanopolystyreneexposed tbh-1(RNAi) nematodes compared with nanopolystyrene-exposed wild-type nematodes (Fig. 3c, d). In contrast, RNAi knockdown of cat-2 significantly inhibited the nanopolystyrene toxicity in inducing intestinal ROS production and in decreasing locomotion behavior (Fig. 3c, d). Therefore, both TBH-1 and CAT-2 were involved in the regulation of response to nanopolystyrene.
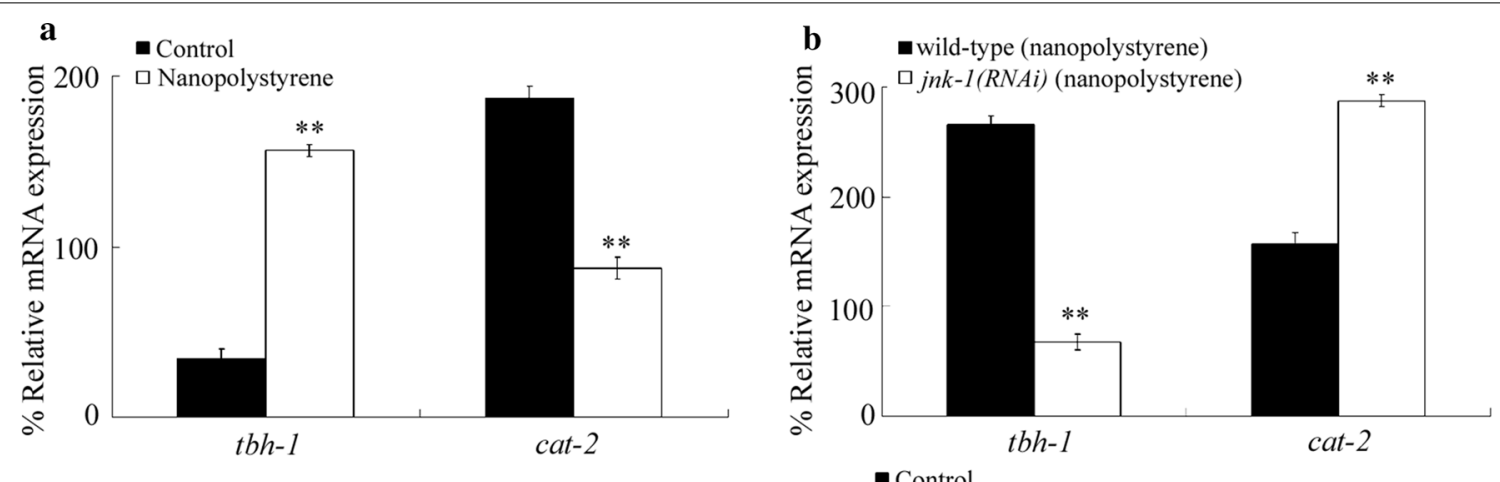

c
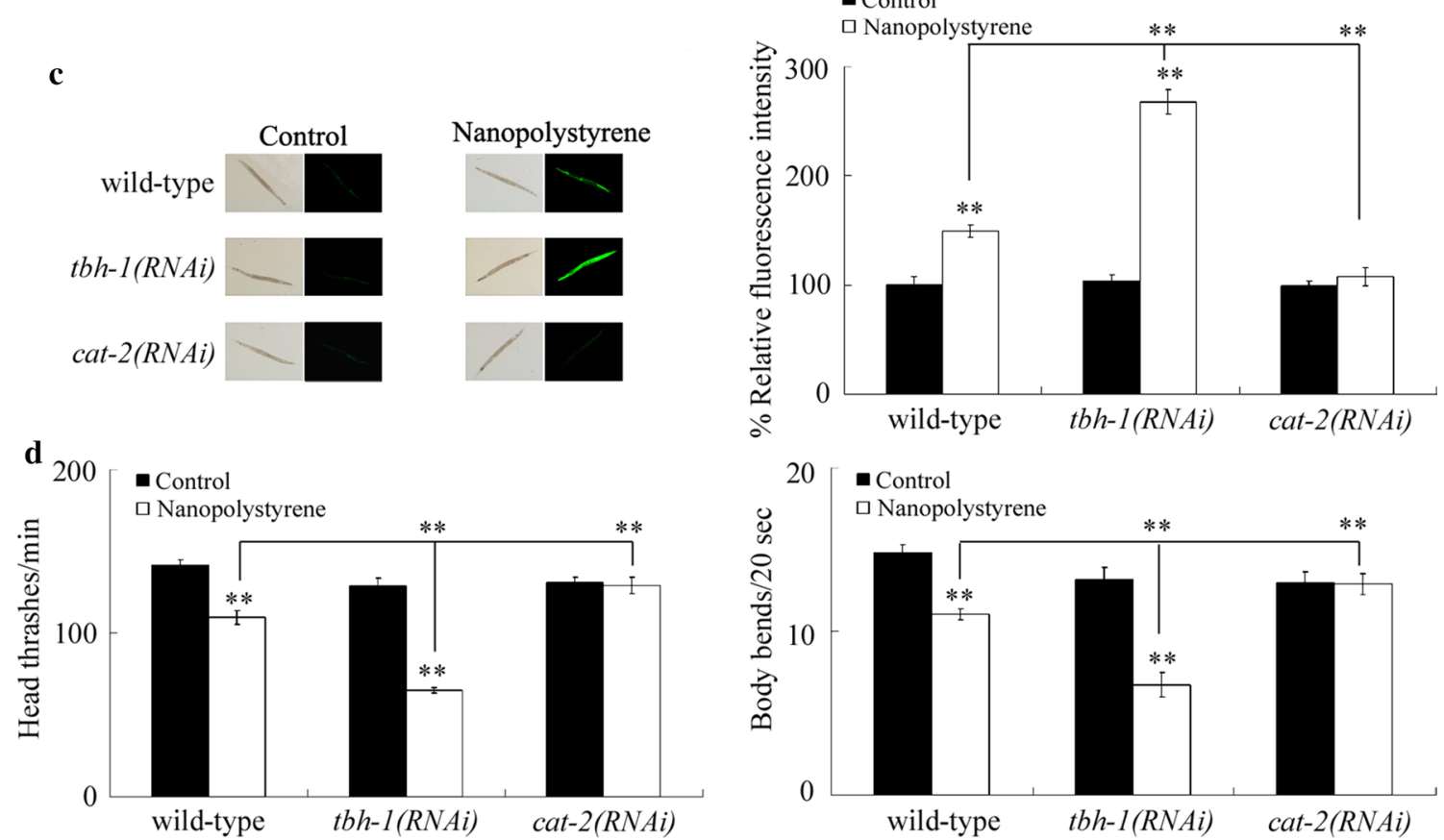

Fig. 3 Involvement of TBH-1 and CAT-2 in the control of response to nanopolystyrene. a Effect of nanopolystyrene exposure on expressions of tbh-1 and cat-2. Bars represent mean $\pm S D .{ }^{* *} P<0.01$ vs. control. b Effect of jnk-1 RNAi knockdown on expressions of tbh-1 and cat-2 in nanopolystyrene-exposed nematodes. Bars represent mean \pm SD. ${ }^{* *} P<0.01$ vs. wild-type. $\mathbf{c}$ Effect of tbh-1 or cat-2 RNAi knockdown on nanopolystyrene toxicity in inducing intestinal ROS production. Bars represent mean $\pm S D$. ${ }^{* *} P<0.01$ vs. control (if not specially indicated). $\mathbf{d}$ Effect of tbh-1 or cat-2 RNAi knockdown on nanopolystyrene toxicity in decreasing locomotion behavior. Bars represent mean \pm SD. ${ }^{* *} P<0.01$ vs. control (if not specially indicated). Exposure concentration of nanopolystyrene was $1 \mu \mathrm{g} / \mathrm{L}$. Exposure was performed from L1 larvae to adult day-3 


\section{Genetic interaction between JNK-1 and TBH-1 or CAT-2 in regulating the response to nanopolystyrene}

To determine the genetic interaction between JNK-1 and $\mathrm{TBH}-1$ in regulating the response to nanopolystyrene, we performed RNAi knockdown of $t b h-1$ in nematodes overexpressing neuronal JNK-1. We observed that RNAi knockdown of $t b h-1$ could induce the significant ROS production and decrease in locomotion behavior in nanopolystyrene-exposed nematodes overexpressing neuronal JNK-1 (Additional file 1: Figure S5), which suggested that RNAi knockdown of $t b h-1$ could suppress the resistance of nematodes overexpressing neuronal JNK-1 to nanopolystyrene toxicity. Meanwhile, we observed that RNAi knockdown of cat-2 inhibited the induction of ROS production and the decrease in locomotion behavior in nanopolystyrene-exposed jnk-1(RNAi) nematodes (Additional file 1: Figure S5), which suggested that RNAi knockdown of cat-2 could suppress the susceptibility of jnk-1(RNAi) nematodes to nanopolystyrene toxicity.

\section{Effect of RNAi knockdown of cat-2 or tbh-1 on expression of intestinal genes encoding the corresponding neurotransmitter receptors in nanopolystyrene-exposed nematodes}

To determine the octopamine- and the dopamine-mediated communication between neurons and intestine, we next examined the effect of RNAi knockdown of cat- 2 or $t b h-1$ on expression of intestinal genes encoding the corresponding neurotransmitter receptors in nanopolystyrene-exposed nematodes. In nanopolystyrene-exposed nematodes, RNAi knockdown of cat-2 could significantly increase the expression of intestinal dop-1 (Fig. 4a). Meanwhile, in nanopolystyrene-exposed nematodes, RNAi knockdown of $t b h-1$ could significantly decrease the expression of intestinal ser- 6 and increase the expression of intestinal octr-1 (Fig. 4b).

\section{Effect of intestinal RNAi knockdown of dop-1, octr-1, or ser-6 on expression of genes encoding p38 MAPK and insulin signaling pathways in nanopolystyrene-exposed nematodes}

Our previous studies have indicated that p38 MAPK and insulin signaling pathways acted in the intestine to regulate the response to nanopolystyrene [33, 34]. In nematodes, $p m k-1$ encodes the p38 MAPK in p38 MAPK signaling pathway, and daf-16 encodes the FOXO transcriptional factor in insulin signaling pathway. Recently, it has been further shown that the signaling cascade of $m d t-15-s b p-1$ encoding the lipid metabolic signaling acted downstream of p38 MAPK signaling to regulate the response to nanopolystyrene [35]. In the intestine, we further examined the effect of RNAi knockdown of dop1 , octr-1, or ser- 6 on expression of genes encoding p38
MAPK and insulin signaling pathways in nanopolystyrene-exposed nematodes. In nanopolystyrene-exposed nematodes, intestinal RNAi knockdown of dop-1 could significantly decrease the expressions of $p m k-1$, $m d t$ 15 , and $s b p-1$ (Fig. 4c). In contrast, in nanopolystyreneexposed nematodes, intestinal RNAi knockdown of octr-1 or ser-6 did not affect the expressions of pmk-1, $m d t-15$, and $s b p-1$ (Fig. 4c). In addition, in nanopolystyrene-exposed nematodes, intestinal RNAi knockdown of dop-1, octr-1 or ser-6 did not influence the expression of daf-16 (Fig. 4d).

\section{Discussion}

In nematodes, MEK-1 and JKK-1 act as co-activators of JNK/JNK-1 [21, 24, 40]. In this study, we observed that exposure to nanopolystyrene $(100 \mathrm{~nm})$ in the range of $\mu \mathrm{g} / \mathrm{L}$ could significantly increase the expressions of $j k k-1, m e k-1$, and jnk-1 (Fig. 1a). Meanwhile, using intestinal ROS production and locomotion behavior as the endpoints, RNAi knockdown of jkk-1, mek-1, or jnk-1 induced a susceptibility to nanopolystyrene toxicity (Fig. 1b, c). Therefore, the alteration in genes encoding the JNK MAPK signaling pathway induced by nanopolystyrene in the range of $\mu \mathrm{g} / \mathrm{L}$ mediated a protective response to nanopolystyrene. Similarly, the alteration in genes encoding insulin and p38 MAPK signaling pathways induced by nanopolystyrene in the range of $\mu \mathrm{g} / \mathrm{L}$ also mediated a protective response to nanopolystyrene in nematodes [33-35].

Some reports have predicted that the range of environmental concentrations for nanoplastics $(1-100 \mathrm{~nm})$ is $\leq 1 \mu \mathrm{g} / \mathrm{L}[29,30]$. In this study, we observed that exposure to $100 \mathrm{~nm}$ nanopolystyrene $(1 \mu \mathrm{g} / \mathrm{L})$ could only increase the $j n k-1$ expression in JNK MAPK signaling pathway (Fig. 1a). Our previous study has also indicated that exposure to $100 \mathrm{~nm}$ nanopolystyrene $(1 \mu \mathrm{g} / \mathrm{L})$ could only increase the $p m k-1$ expression in p38 MAPK signaling pathway [34]. That is, exposure to nanopolystyrene at predicted environmental concentration may only be able to affect the expression of gene encoding MAPK in JNK MAPK or p38 MAPK signaling pathway in nematodes.

Considering the fact that $j n k-1$ is expressed in the neurons [21, 24, 40], the JNK MAPK signaling provided an important molecular basis for neuronal response to nanopolystyrene. In C. elegans, there are three MAPK signaling pathways (p38, JNK, and ERK signaling pathways) $[21,24]$. p38 MAPK signaling pathway acted in the intestine to regulate the response to nanopolystyrene in nematodes $[34,35]$. These observations suggest that different MAPK signaling pathways can provide the molecular basis for different tissues in response to nanopolystyrene.

In the neurons, SNB-1/synaptobrevin was identified as the downstream target of JNK-1/JNK during the 

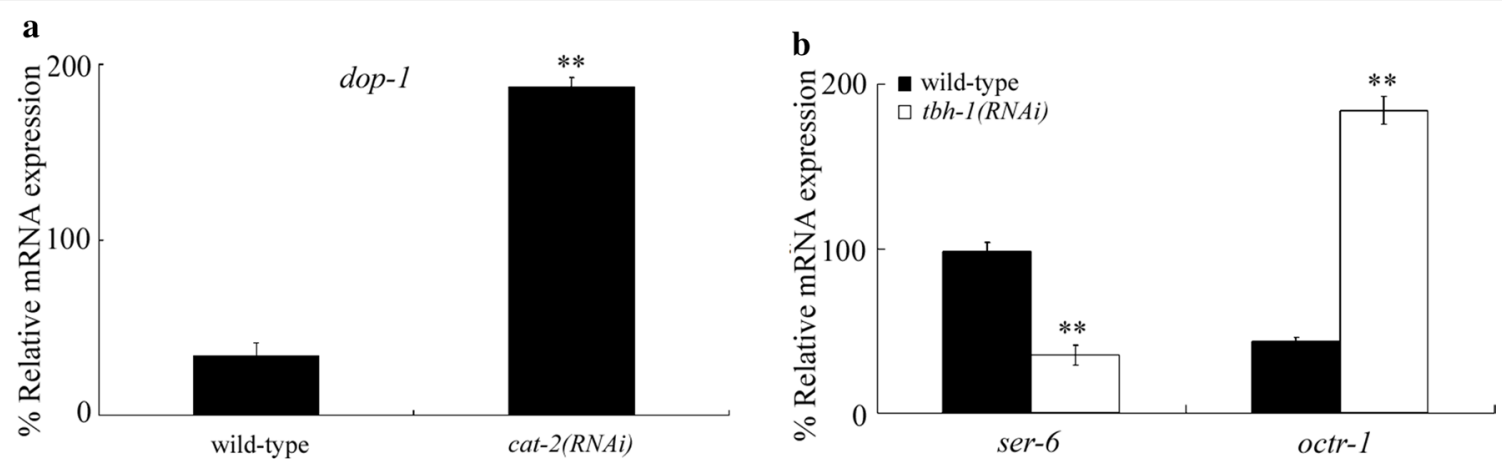

c

d
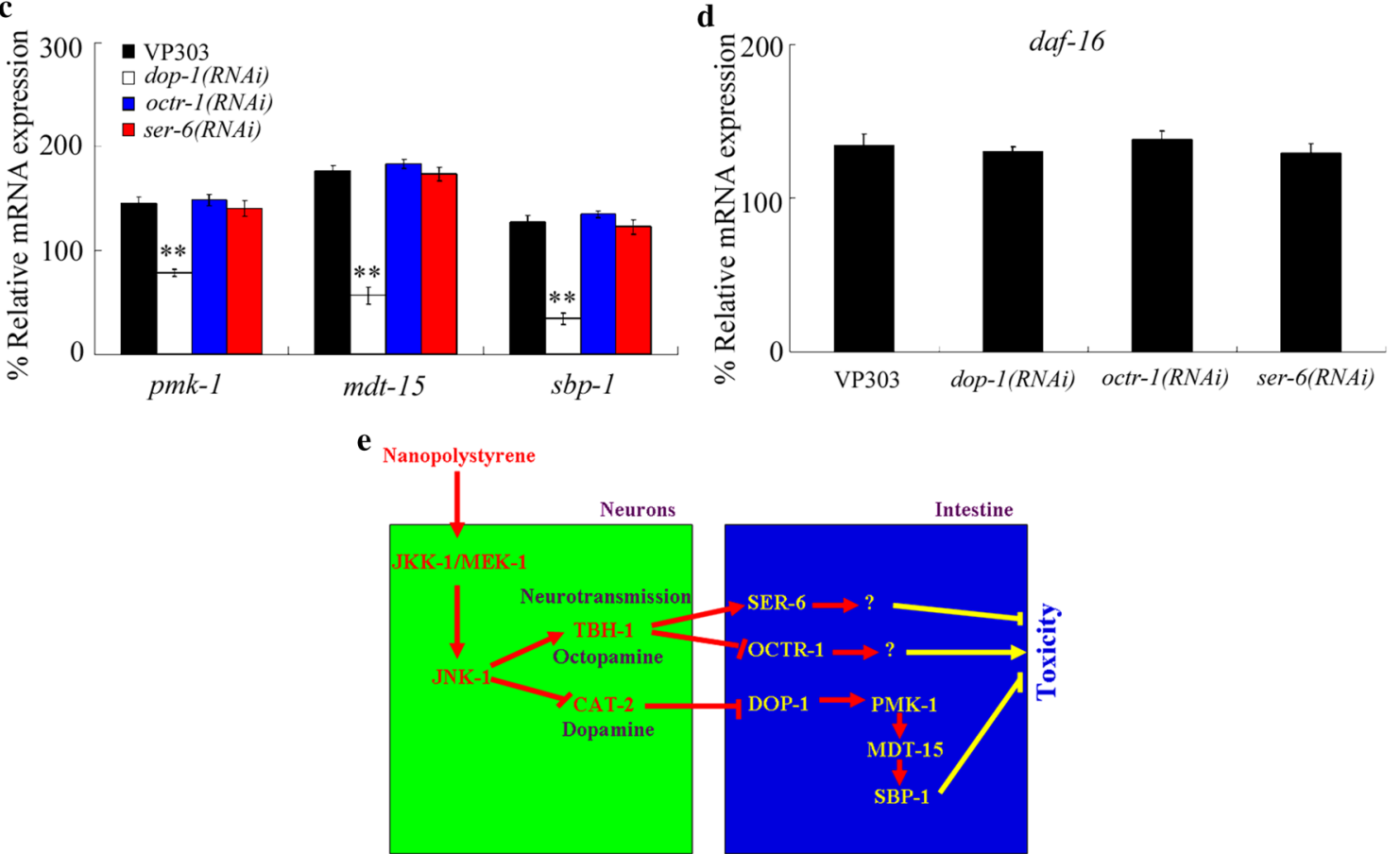

Fig. 4 Involvement of octopamine and dopamine signals in the regulation of response to nanopolystyrene. a Effect of RNAi knockdown of cat-2 on expression of intestinal dop-1 in nanopolystyrene-exposed nematodes. qRT-PCR was performed in isolated intact intestines $(n=40)$. Exposure concentration of nanopolystyrene was $1 \mu \mathrm{g} / \mathrm{L}$. Exposure was performed from $\mathrm{L} 1$ larvae to adult day-3. Bars represent mean $\pm S D .{ }^{* *} P<0.01$ vs. wild-type. $\mathbf{b}$ Effect of RNAi knockdown of tbh-1 on expressions of intestinal ser- 6 and octr- 1 in nanopolystyrene-exposed nematodes. qRT-PCR was performed in isolated intact intestines $(n=40)$. Exposure concentration of nanopolystyrene was $1 \mu \mathrm{g} / \mathrm{L}$. Exposure was performed from $\mathrm{L} 1$ larvae to adult day-3. Bars represent mean $\pm S D .{ }^{* *} P<0.01$ vs. wild-type. $\mathbf{c}$ Effect of intestinal RNAi knockdown of dop-1, octr-1, or ser- 6 on expressions of pmk-1, mdt-15, or sbp-1 in nanopolystyrene-exposed nematodes. Exposure concentration of nanopolystyrene was $1 \mu \mathrm{g} / \mathrm{L}$. Exposure was performed from L1 larvae to adult day-3. Bars represent mean $\pm S D$. ${ }^{* *} P<0.01$ vs. VP303. d Effect of intestinal RNAi knockdown of dop-1, octr-1, or ser-6 on expression of daf-16 in nanopolystyrene-exposed nematodes. Exposure concentration of nanopolystyrene was $1 \mu \mathrm{g} / \mathrm{L}$. Exposure was performed from L1 larvae to adult day-3. Bars represent mean $\pm S D$. ${ }^{*} P<0.01$ vs. VP303. e A diagram showing the molecular basis of neuronal JNK MAPK signaling in regulating the response to nanopolystyrene in nematodes

regulation of response to nanopolystyrene (Additional file 1: Figure S3). The vesicle-associated protein SNB-1 mediates the neurotransmitter release [48], which suggested that the JNK MAPK signaling may regulate the response to nanopolystyrene by potentially modulating the neurotransmitter signals in the neurons (Fig. 4e). In nematodes, it was also reported that the PKC-1 governing the neurotransmitter release also acted as the downstream target of neuronal NLG-1 signaling to regulate the response to $\mathrm{GO}[21,24,53]$. In the neurons, we further provide the evidence to indicate the role of JNK MAPK signaling in modulating the TBH-1-mediated octopamine signal and the CAT-2-mediated dopamine signal in nanopolystyrene-exposed nematodes (Fig. 3 and Additional file 1: Figure S5), which supported the function of neuronal JNK MAPK signaling raised above. 
Nevertheless, the identification of TBH-1-mediated octopamine signal and CAT-2-mediated dopamine signal in nanopolystyrene-exposed nematodes was based on the JNK MAPK signaling-activated communication between the neurons and the intestine. That is, we still do not exclude the possibility that the other possible neurotransmitter signals may also be modulated by neuronal JNK MAPK signaling in nanopolystyrene-exposed nematodes.

In nematodes, TBH-1 and CAT-2 regulated the response to nanopolystyrene by affecting the functions of corresponding intestinal octopamine receptors (SER-6 and OCTR-1) and intestinal dopamine receptor (DOP-1) (Figs. 2 and 4a, b). Especially, RNAi knockdown of $t b h-1$ could decrease the ser- 6 expression and increase the octr1 expression in nanopolystyrene-exposed nematodes (Fig. 4b), which suggested that the neuronal TBH-1 can activate both positive and negative regulators in the intestine to regulate the response to nanopolystyrene. These observations demonstrated that the neuronal TBH-1 and CAT-2 and their corresponding intestinal octopamine and dopamine receptors established a communication between the neurons and the intestine required for the control of response to nanopolystyrene in nematodes (Fig. 4e). These observations suggested an important molecular basis for the communication between the neurons and the intestine in nanopolystyrene-exposed nematodes.

So far, in the intestine, two signaling pathways (insulin and p38 MAPK signaling pathways) have been identified to be involved in the control of response to nanopolystyrene $[33-35,54]$. In the intestine, we found that the DOP-1 regulated the response to nanopolystyrene by activating the downstream signaling cascade in p38 MAPK signaling pathway (Fig. 4c). The downstream targets of SER- 6 and OCTR-1 in the intestine during the control of response to nanopolystyrene are still unclear (Fig. 4e). Meanwhile, we found that DOP-1, SER-6, and OCTR-1 were not the upregulators of intestinal DAF16 in the insulin signaling pathway in the regulation of response to nanopolystyrene (Fig. 4d). In the intestine, the insulin receptor DAF-2 could regulate the response to nanopolystyrene by suppressing the function of DAF16 in nematodes [33].

\section{Conclusions}

We here employed $C$. elegans as an animal model to investigate the role of neuronal JNK MAPK signaling in response to nanopolystyrene and the underlying mechanism. In nematodes, exposure to $100 \mathrm{~nm}$ nanopolystyrene in the range of $\mu \mathrm{g} / \mathrm{L}$ induced the increase of genes encoding JNK MAPK signaling pathway, which mediated a protective response to nanopolystyrene. This activated JNK MAPK signaling modulated the TBH-1-mediated octopamine and CAT-2-mediated dopamine signals in neurons in nanopolystyrene-exposed nematodes. TBH-1 regulated the response to nanopolystyrene by affecting its intestinal receptors of SER-6 and OCTR-1, and CAT-2 regulated the response to nanopolystyrene by affecting its intestinal receptor of DOP-1. In the intestine, dopamine receptor further regulated the response to nanopolystyrene by activating the $\mathrm{p} 38$ MAPK signaling pathway. Our results highlight the importance of octopamine- and dopaminemediated neuron-intestine communication activated by neuronal JNK MAPK signaling in regulating the response to nanopolystyrene in organisms.

\section{Supplementary information}

Supplementary information accompanies this paper at https://doi. org/10.1186/s12302-020-00331-8.

Additional file 1: Figure S1. RNAi efficiency confirmed by qRT-PCR. $\left\llcorner 4440\right.$, empty vector. Bars represent mean $\pm S D$. ${ }^{* *} P<0.01$ vs. L4440. Figure S2. Properties of nanopolystyrene. Figure S3. Identification of targets of JNK-1 in regulating the response to nanopolystyrene. Figure S4. Effect of snb-1 RNAi knockdown on nanopolystyrene toxicity in nematodes overexpressing neuronal JNK-1. Figure S5. Genetic interaction between JNK-1 and $\mathrm{TBH}-1$ or $\mathrm{CAT}-2$ in regulating the nanopolystyrene toxicity in inducing intestinal ROS production. Table S1. Primer information for qRT-PCR. Table S2. Primer information for vector constructions.

\section{Abbreviations}

ROS: Reactive oxygen species; MAPK: Mitogen-activated protein kinase; JNK: c-Jun N-terminal kinase; TEM: Transmission electron microscopy; DLS: Dynamic light scattering; CFUs: Colony-forming units; NGM: Nematode growth media; qRT-PCR: Quantitative real-time polymerase chain reaction; RNAi: RNA interference; IPTG: Isopropyl 1-thio- $\beta$-D-galactopyranoside; ANOVA: Analysis of variance.

\section{Acknowledgements}

Not applicable.

\section{Authors' contributions}

$M Q, D L, Y Z$, and $Y Y$ performed the experiments. DW designed the experiment and wrote the manuscript. All authors read and approved the final manuscript.

\section{Funding}

This work was supported by the grant from Basic and Advanced Research Project of Chongqing CSTC (No. cstc2019jcyj-msxmX0533).

Availability of data and materials

The data supporting the conclusions of this article are included within the article and supporting materials.

Ethics approval and consent to participate Not applicable.

Consent for publication

Not applicable.

\section{Competing interests}

The authors declare that they have no competing interests.

Received: 11 February 2020 Accepted: 23 March 2020

Published online: 07 April 2020 


\section{References}

1. Yang D, Shi H, Li L, Li J, Jabeen K, Kolandhasamy P (2015) Microplastic pollution in table salts from China. Environ Sci Technol 49:13622-13627

2. Su L, Xue Y, Li L, Yang D, Kolandhasamy P, Li D, Shi H (2016) Microplastics in Taihu Lake, China. Environ Pollut 216:711-719

3. Wang W, Ndungu AW, Li Z, Wang J (2017) Microplastics pollution in inland freshwaters of China: a case study in urban surface waters of Wuhan, China. Sci Total Environ 575:1369-1374

4. Zhang G, Liu Y (2018) The distribution of microplastics in soil aggregate fractions in southwestern China. Sci Total Environ 642:12-20

5. Cai L, Wang J, Peng J, Tan Z, Zhan Z, Tan X, Chen Q (2017) Characteristic of microplastics in the atmospheric fallout from Dongguan city, China: preliminary research and first evidence. Environ Sci Pollut Res Int 24:24928-24935

6. Chae Y, An YJ (2017) Effects of micro- and nanoplastics on aquatic ecosystems: current research trends and perspectives. Marine Pollut Bull 124:624-632

7. Cheung PK, Fok L (2016) Evidence of microbeads from personal care product contaminating the sea. Mar Pollut Bull 109:582-585

8. Mattsson K, Hansson LA, Cedervall T (2015) Nano-plastics in the aquatic environment. Environ Sci Process Impacts 17:1712-1721

9. Li L, Geng S, Wu C, Song K, Sun F, Visvanathan C, Xie F, Wang Q (2019) Microplastics contamination in different trophic state lakes along the middle and lower reaches of Yangtze River basin. Environ Pollut 254:112951

10. Xiong X, Wu C, Elser JJ, Mei Z, Hao Y (2019) Occurrence and fate of microplastic debris in middle and lower reaches of the Yangtze River from inland to the sea. Sci Total Environ 659:66-73

11. Della Torre C, Bergami E, Salvati A, Faleri C, Cirino P, Dawson KA, Corsi (2014) Accumulation and embryotoxicity of polystyrene nanoparticles at early stage of development of sea urchin embryos Paracentrotus lividus. Environ Sci Technol 48:12302-12311

12. Jeong C, Won E, Kang H, Lee M, Hwang D, Hwang U, Zhou B, Souissi S, Lee S, Lee J (2016) Microplastic size-dependent toxicity, oxidative stress induction, and p-JNK and p-p38 activation in the monogonont rotifer (Brachionus koreanus). Environ Sci Technol 50:8849-8857

13. Ma Y, Huang A, Cao S, Sun F, Wang L, Guo H, Ji R (2016) Effects of nanoplastics and microplastics on toxicity, bioaccumulation, and environmental fate of phenanthrene in fresh water. Environ Pollut 219:166-173

14. Rist S, Baun A, Hartmann NB (2017) Ingestion of micro- and nanoplastics in Daphnia magna quantification of body burdens and assessment of feeding rates and reproduction. Environ Pollut 228:398-407

15. Chen Q, Gundlach M, Yang S, Jiang J, Velki M, Yin D, Hollert H (2017) Quantitative investigation of the mechanisms of microplastics and nanoplastics toward zebrafish larvae locomotor activity. Sci Total Environ 584-585:1022-1031

16. Ziajahromi S, Kumar A, Neale PA, Leusch FDL (2018) Environmentally relevant concentrations of polyethylene microplastics negatively impact the survival, growth and emergence of sediment-dwelling invertebrates. Environ Pollut 236:425-431

17. Pitt JA, Trevisan R, Massarsky A, Kozal JS, Levin ED, Di Giulio RT (2018) Maternal transfer of nanoplastics to offspring in zebrafish (Danio rerio): a case study with nanopolystyrene. Sci Total Environ 643:324-334

18. Brenner S (1974) The genetics of Caenorhabditis elegans. Genetics 77:71-94

19. Leung MC, Williams PL, Benedetto A, Au C, Helmcke KJ, Aschner M, Meyer JN (2008) Caenorhabditis elegans: an emerging model in biomedical and environmental toxicology. Toxicol Sci 106:5-28

20. Liu HL, Guo DQ, Kong Y, Rui Q, Wang DY (2019) Damage on functional state of intestinal barrier by microgravity stress in nematode Caenorhabditis elegans. Ecotoxicol Environ Saf 183:109554

21. Wang DY (2019) Target organ toxicology in Caenorhabditis elegans. Springer Nature, Singapore

22. Zhao YL, Chen H, Yang YH, Wu QL, Wang DY (2020) Graphene oxide disrupts the protein-protein interaction between Neuroligin/NLG-1 and DLG-1 or MAGI-1 in nematode Caenorhabditis elegans. Sci Total Environ 700:134492

23. Rui Q, Dong SS, Jiang WK, Wang DY (2019) Response of canonical Wnt/ $\beta$-catenin signaling pathway in the intestine to microgravity stress in Caenorhabditis elegans. Ecotoxicol Environ Saf 186:109782
24. Wang DY (2019) Molecular toxicology in Caenorhabditis elegans. Springer Nature, Singapore

25. Hanna SK, Montoro Bustos AR, Peterson AW, Reipa V, Scanlan LD, Hosbas Coskun S, Cho TJ, Johnson ME, Hackley VA, Nelson BC, Winchester MR, Elliott JT, Petersen EJ (2018) Agglomeration of Escherichia coli with positively charged nanoparticles can lead to artifacts in a standard Caenorhabditis elegans toxicity assay. Environ Sci Technol 52:5968-5978

26. Lei L, Wu S, Lu S, Liu M, Song Y, Fu Z, Shi H, Raley-Susman KM, He D (2018) Microplastic particles cause intestinal damage and other adverse effects in zebrafish Danio rerio and nematode Caenorhabditis elegans. Sci Total Environ 619-620:1-8

27. Qu M, Luo LB, Yang YH, Kong Y, Wang DY (2019) Nanopolystyreneinduced microRNAs response in Caenorhabditis elegans after long-term and lose-dose exposure. Sci Total Environ 697:134131

28. Qu M, Qiu YX, Kong Y, Wang DY (2019) Amino modification enhances reproductive toxicity of nanopolystyrene on gonad development and reproductive capacity in nematode Caenorhabditis elegans. Environ Pollut 254:112978

29. Lenz R, Enders K, Nielsen TG (2016) Microplastic exposure studies should be environmentally realistic. Proc Natl Acad Sci USA 113:E4121-E4122

30. Al-Sid-Cheikh M, Rowland S, Stevenson K, Rouleau C, Henry TB, Thompson RC (2018) Uptake, whole-body distribution, and depuration of nanoplastics by the scallop pecten maximus at environmentally realistic concentrations. Environ Sci Technol 52:14480-14486

31. Qu M, Zhao YL, Zhao YY, Rui Q, Kong Y, Wang DY (2019) Identification of long non-coding RNAs in response to nanopolystyrene in Caenorhabditis elegans after long-term and low-dose exposure. Environ Pollut 255:113137

32. Qu M, Nida A, Kong Y, Du HH, Xiao GS, Wang DY (2019) Nanopolystyrene at predicted environmental concentration enhances microcystin-LR toxicity by inducing intestinal damage in Caenorhabditis elegans. Ecotoxicol Environ Saf 183:109568

33. Shao HM, Han ZY, Krasteva N, Wang DY (2019) Identification of signaling cascade in the insulin signaling pathway in response to nanopolystyrene particles. Nanotoxicology 13:174-188

34. Qu M, Liu YQ, Xu KN, Wang DY (2019) Activation of p38 MAPK signalingmediated endoplasmic reticulum unfolded protein response by nanopolystyrene particles. Adv Biosys 3:1800325

35. Yang YH, Shao HM, Wu QL, Wang DY (2020) Lipid metabolic response to polystyrene particles in nematode Caenorhabditis elegans. Environ Pollut 256:113439

36. Koga M, Zwaal R, Guan KL, Avery L, Ohshima Y (2000) A Caenorhabditis elegans MAP kinase kinase, MEK-1, is involved in stress responses. EMBO J 19:5148-5156

37. Wagner EF, Nebreda AR (2009) Signal integration by JNK and p38 MAPK pathways in cancer development. Nat Rev Cancer 3:537-549

38. Mizuno T, Hisamoto N, Terada T, Kondo T, Adachi M, Nishida E, Kim DH, Ausubel FM, Matsumoto K (2004) The Caenorhabditis elegans MAPK phosphatase VHP-1 mediates a novel JNK-like signaling pathway in stress response. EMBO J 11:2226-2234

39. Zhao YL, Wu QL, Wang DY (2015) A microRNAs-mRNAs network involved in the control of graphene oxide toxicity in Caenorhabditis elegans. RSC Adv 5:92394-92405

40. Kawasaki M, Hisamoto $N$, lino Y, Yamamoto M, Ninomiya-Tsuji J, Matsumoto K (2014) A Caenorhabditis elegans JNK signal transduction pathway regulates coordinated movement via type-D GABAergic motor neurons. EMBO J 18:3604-3615

41. Liu PD, Shao HM, Kong Y, Wang DY (2020) Effect of graphene oxide exposure on intestinal Wnt signaling in nematode Caenorhabditis elegans. J Environ Sci 88:200-208

42. Wang S, Zhao Y, Wu L, Tang M, Su C, Hei TK, Yu Z (2007) Induction of germline cell cycle arrest and apoptosis by sodium arsenite in Caenorhabditis elegans. Chem Res Toxicol 29:181-186

43. Cheng XK, Dong SS, Chen D, Rui Q, Guo JJ, Wang DY, Jiang JD (2020) Potential of esterase DmtH in transforming plastic additive dimethyl terephthalate to less toxic mono-methyl terephthalate. Ecotoxicol Environ Saf 187:109848

44. Zhao $Y L$, Jin L, Wang $Y$, Kong $Y$, Wang DY (2019) Prolonged exposure to multi-walled carbon nanotubes dysregulates intestinal mir-35 and its direct target MAB-3 in nematode Caenorhabditis elegans. Sci Rep 9:12144

45. Mello C, Fire A (1995) DNA transformation. method Cell Biol 48:451-482 
46. Byrd DT, Kawasaki M, Walcoff M, Hisamoto N, Matsumoto K, Jin Y (2001) UNC-16, a JNK-signaling scaffold protein, regulates vesicle transport in C. elegans. Neuron 32:787-800

47. Neumann-Haefelin E, Qi W, Finkbeiner E, Walz G, Baumeister R, Hertweck M (2008) SHC-1/p52Shc targets the insulin/IGF-1 and JNK signaling pathways to modulate life span and stress response in C. elegans. Genes Dev 22:2721-2735

48. Nonet ML, Saifee O, Zhao H, Rand JB, Wei L (1998) Synaptic transmission deficits in Caenorhabditis elegans synaptobrevin mutants. J Neurosci 18:70-80

49. Zhao L, Qu M, Wong G, Wang DY (2017) Transgenerational toxicity of nanopolystyrene particles in the range of $\mu \mathrm{g} / \mathrm{L}$ in nematode Caenorhabditis elegans. Environ Sci Nano 4:2356-2366

50. Espelt MV, Estevez AY, Yin X, Strange K (2005) Oscillatory Ca ${ }^{2+}$ signaling in the isolated Caenorhabditis elegans intestine. Role of the inositol-1,4,5trisphosphate receptor and phospholipases $C \beta$ and $\gamma$. J Gen Physiol 126:379-392
51. Alkema MJ, Hunter-Ensor M, Ringstad N, Horvitz HR (2005) Tyramine functions independently of octopamine in the Caenorhabditis elegans nervous system. Neuron 46:247-260

52. Sawin ER, Ranganathan R, Horvitz HR (2000) C. elegans locomotory rate is modulated by the environment through a dopaminergic pathway and by experience through a serotonergic pathway. Neuron 26:619-631

53. Chen H, Li HR, Wang DY (2017) Graphene oxide dysregulates Neuroligin/ NLG-1-mediated molecular signaling in interneurons in Caenorhabditis elegans. Sci Rep 7:41655

54. Liu HL, Shao HM, Guo ZJ, Wang DY (2020) Nanopolystyrene exposure activates a fat metabolism related signaling-mediated protective response in Caenorhabditis elegans. Nanolmpacts 17:100204

\section{Publisher's Note}

Springer Nature remains neutral with regard to jurisdictional claims in published maps and institutional affiliations.

\section{Submit your manuscript to a SpringerOpen ${ }^{\circ}$ journal and benefit from:}

- Convenient online submission

- Rigorous peer review

- Open access: articles freely available online

- High visibility within the field

- Retaining the copyright to your article

Submit your next manuscript at $\boldsymbol{\nabla}$ springeropen.com 